\title{
A WAVE EQUATION WITH A POSSIBLY JUMPING NONLINEARITY
}

\author{
J. R. WARD, JR. ${ }^{1}$
}

\begin{abstract}
Existence of a doubly periodic solution to a forced semilinear wave equation is established. The nonlinearity may "jump" across any finite number of eigenvalues of finite multiplicity.
\end{abstract}

1. Introduction. Let $J=[0,2 \pi] \times[0,2 \pi]$ and let $f: J \times \mathbf{R} \rightarrow \mathbf{R},(t, x, s) \mapsto$ $f(t, x, s)$, be a function satisfying the Carathéodory conditions. Assume there is a number $A>0$ and a function $B \in L^{2}(J)$ such that for each $s \in \mathbf{R}$ and $(t, x) \in J$ we have

$$
|f(t, x, s)| \leq A|s|+B(t, x) .
$$

Let $h \in L^{2}(J)$. We consider the existence (in the weak sense) of solutions $2 \pi$ periodic in each of $x$ and $t$ for the semilinear wave equation

$$
u_{t t}-u_{x x}-f(t, x, u)=h(t, x) .
$$

By a weak solution to the doubly $2 \pi$-periodic problem for (1.2) is meant a $u \in$ $L^{2}(J)$ such that

$$
\begin{aligned}
\int_{J} u( & (t, x)\left[v_{t t}(t, x)-v_{x x}(t, x)\right] d t d x \\
= & \int_{J}[f(t, x, u(t, x))+h(t, x)] v(t, x) d t d x
\end{aligned}
$$

for every $v \in C^{2}(J)$ satisfying the boundary conditions

$$
\begin{array}{ll}
v(t, 0)-v(t, 2 \pi)=v_{x}(t, 0)-v_{x}(t, 2 \pi)=0 & (t \in[0,2 \pi]), \\
v(0, x)-v(2 \pi, x)=v_{t}(0, x)-v_{t}(2 \pi, x)=0 & (x \in[0,2 \pi]) .
\end{array}
$$

If $\lambda \in \mathbf{R}$ the doubly $2 \pi$-periodic problem for

$$
u_{t t}-u_{x x}-\lambda u=h(t, x)
$$

has a unique weak solution for every $h \in L^{2}(J)$ if and only if $\lambda \notin \Sigma$, where

$$
\Sigma=\left\{n^{2}-m^{2}:(m, n) \in Z \times Z\right\}=\left\{\ldots, \lambda_{-2}, \lambda_{-1}, \lambda_{0}=0, \lambda_{1}, \lambda_{2}, \ldots\right\}
$$

and $Z$ denotes the integers.

Received by the editors October 4, 1983.

1980 Mathematics Subject Classification. Primary 35B10, 35L05, 47H15.

Key words and phrases. Nonlinear wave equation, periodic solution.

${ }^{1}$ This work was done while the author was visiting the Université Catholique de Louvain, Louvain-la-Neuve, Belgium. 
Existence results for (1.2) with any of the usual boundary conditions (e.g., doubly periodic, periodic-Dirichlet) usually require $f$ to be monotone in $s$. The monotonicity enables one to work around difficulties created by the infinite multiplicity of the eigenvalue $\lambda_{0}=0$.

It is known that, with our boundary conditions, (1.2) has a solution for each $h \in H$ if $f$ is monotone in $s$ and is asymptotically between (and bounded away from) two successive eigenvalues (Mawhin [M.1]). or if $f$ is monotone in $s$ and "jumps" (asymptotically, going from $-\infty$ to $+\infty$ ) from one eigenvalue to the next, or to the one below (provided neither is $\lambda_{0}=0$ ) (Willem [Wi]).

These results have been recently unified and generalized to include nonuniformities in the avoidance of $\Sigma$ (Mawhin and Ward [M-W.1, M-W.2]). Here we show that $f$ may jump across arbitrarily many eigenvalues of finite multiplicity and (1.3) may still be solvable for all $h \in H$.

2. Statement of results. The following is our main result.

THEOREM 1. Let $f: J \times \mathbf{R} \rightarrow \mathbf{R}$ satisfy the Carathéodory conditions and (1.1). Let $h \in L^{2}(J)$ and suppose:

(c1) $f(t, x, s)$ is monotone nondecreasing in $s$ for each $(t, x) \in J$.

(c2) There is a number $\alpha>0$ and a function $\beta \in H$ such that

$$
|f(t, x, s)| \leq f(t, x, s)+\alpha|s|+\beta(t, x)
$$

for all $(t, x, s) \in J \times \mathbf{R}$.

(c3) There is a number $\eta_{0}>0$ such that $0<\eta_{0} \leq \underline{\lim }_{|s| \rightarrow \infty} s^{-1} f(t, x, s)$, uniformly in $(t, x) \in J$.

Then there is a number $\alpha_{0}>0$ such that a weak solution to the doubly $2 \pi$-periodic problem for (1.2) exists whenever $\alpha<\alpha_{0}$.

REMARK 1. One may instead assume $f(t, x, s)$ is nonincreasing in $s$ and the existence of $\eta_{0}<0$ with $0>\eta_{0} \geq \varlimsup_{|s| \rightarrow \infty} s^{-1} f(t, x, s)$. One may also replace $f$ in $(\mathrm{c} 2)$ by $-f$.

As a corollary we have the following result on jumping nonlinearities. Consider the equation

$$
u_{t t}-u_{x x}+\alpha_{-} u^{-}-\alpha_{+} u^{+}-g(t, x, u)=h(t, x)
$$

where $u^{+}=\max (u, 0), u^{-}=\max (-u, 0)$, and $u=u^{+}-u^{-}$. Suppose $h \in L^{2}(J)$ and $g: J \times \mathbf{R} \rightarrow \mathbf{R}$ satisfies the Carthéodory conditions and (1.1).

Corollary 1. Let $\alpha_{-}$and $\alpha_{+}$be positive numbers. Suppose $f(t, x, s):=$ $-\alpha_{-} s^{-}+\alpha_{+} s^{+}+g(t, x, s)$ is monotone nondecreasing in $s$ and

$$
\lim _{|s| \rightarrow \infty} s^{-1} g(t, x, s)=0,
$$

uniformly for $(t, x) \in s$. Then there is a number $\alpha_{0}>0$ such that (2.1) has a weak doubly $2 \pi$-periodic solution provided $0<\alpha_{-}<\alpha_{0}$ (or $\left.0<\alpha_{+}<\alpha_{0}\right)$. ( $\alpha_{0}$ does not depend on $\alpha_{+}, g$, or $h$.)

REMARK 2. A similar corollary is true with $\alpha_{-}, \alpha_{+}$negative and $f(t, x, s)$ nonincreasing in $s$.

REMARK 3. We note that if $\alpha_{-}<\alpha_{+}$then we may have $] \alpha_{-}, \alpha_{+}[\cap \Sigma \neq \emptyset$. Indeed, the interval $] \alpha_{-}, \alpha_{+}[$may contain any finite number of positive eigenvalues. 
REMARK 4. The corollary may be viewed as a surjectivity result concerning operators of the form $L u+\alpha_{-} u^{-}-\alpha_{+} u^{+}$, where $L$ is the D'Alembertian realized in $L^{2}(J)$ with our boundary conditions.

3. Abstract formulation and proofs. Let $H=L^{2}(J)$ with inner product $(u, v)=\int_{J} u v d t d x$ and corresponding norm $\|\cdot\|$. Each $u \in H$ has a representation as a Fourier series of the form

$$
u=\sum_{(m, n) \in Z \times Z} \alpha_{m n} u_{m n}
$$

where $u_{m n}=e^{i(m t+n x)}$ for $(m, n) \in Z \times Z$ and $\alpha_{m, n} \in \mathbf{C}$ with $\bar{\alpha}_{m, n}=\alpha_{-m,-n}$ to make the sum real.

Let

$$
D(L)=\left\{u \in H: \sum_{(m, n) \in Z^{2}}\left(n^{2}-m^{2}\right)^{2}\left|\alpha_{m n}\right|^{2}<\infty\right\} .
$$

Define $L: D(L) \subseteq H \rightarrow H$ by

$$
L u=\sum_{(m, n) \in Z \times Z}\left(n^{2}-m^{2}\right) \alpha_{m n} u_{m n} \quad \text { for } u \in D(L) .
$$

Let $F: H \rightarrow H$ be the substitution operator defined by $f$. It is known that $u$ is a weak solution of the doubly $2 \pi$-periodic problem for (1.2) if and only if $u \in D(L)$ and

$$
L u-F(u)=h
$$

ProOF OF THEOREM 1. Let $\lambda_{1}$ be the first positive eigenvalue of $L$ (i.e., smallest positive number in $\Sigma$ so that $\left.\lambda_{1}=1\right)$. Choose $\varepsilon_{0}>0$ so that $\varepsilon_{0}<$ $\min \left(\lambda_{1}, \eta_{0}\right)$. For $\left.\lambda \in\right] 0,1[$ consider the family of equations

$$
L u-(1-\lambda) \varepsilon_{0} u-\lambda F(u)=\lambda h .
$$

The operator $\left(L-\varepsilon_{0} I\right)^{-1}$ is not compact. Nevertheless, it follows from a theorem in Willem's paper [Wi] (or see [M.2]) that it suffices to show that all possible solutions of (3.2) are bounded in $H$ independently of $\lambda \in] 0,1[$.

If $(u, \lambda)$ is a solution of $(3.2)$ with $0<\lambda<1$ then

$$
L u-(1-\lambda) \varepsilon_{0} u-\lambda f(t, x, u)=\lambda h(t, x),
$$

and by taking inner products with 1 we derive, since $(L u, 1)=0$,

$$
\lambda \int_{J} f(t, x, u) d t d x=-(1-\lambda) \varepsilon_{0} \int_{J} u d t d x-\lambda \int_{J} h(t, x) d t d x .
$$

Taking absolute values in (3.3) and using (c2) we have a.e. on $J$ :

$$
\begin{aligned}
|L u(t, x)| \leq & (1-\lambda) \varepsilon_{0}|u(t, x)|+\lambda f(t, x, u(t, x)) \\
& +\alpha|u(t, x)|+\beta(t, x)+|h(t, x)| .
\end{aligned}
$$

Integrating over $J$ and using (3.4) we obtain

$$
|L u|_{L^{1}} \leq\left(2 \varepsilon_{0}+\alpha\right)|u|_{L^{1}}+C_{1}
$$

where $C_{1}$ is a constant. 
For $u \in H$ let us write $u=u_{0}+u_{1}$ with $u_{0} \in \operatorname{ker} L$ and $u_{1} \in \operatorname{ker} L^{\perp}=$ Range $L$. It is known (cf., e.g., $[\mathbf{L}$ or $\mathbf{C - H}]$ ) that there is a constant $\mu>0$ such that for $u=u_{0}+u_{1} \in D(L)$,

$$
\left|u_{1}\right|_{L^{\infty}} \leq \mu\left|L u_{1}\right|_{L^{1}} .
$$

Thus for any solution $u$ of (3.2) we have

$$
\left|u_{1}\right|_{L^{\infty}} \leq \mu\left|L u_{1}\right|_{L^{1}} \leq \mu\left(2 \varepsilon_{0}+\alpha\right)|u|_{L^{1}}+\mu C_{1}
$$

and

$$
\left|u_{1}\right|_{L^{\infty}} \leq\left(2 \varepsilon_{0}+\alpha\right) C_{2}\|u\|+C_{3}
$$

for some constants $C_{2}$ and $C_{3}$. Of course, by (3.5) we also have

$$
\left|L u_{1}\right|_{L^{1}} \leq\left(2 \varepsilon_{0}+\alpha\right) C_{4}\|u\|+C_{1} .
$$

Taking the inner product of the expression on each side of (3.2) with $u$ we derive

$$
(1-\lambda) \varepsilon_{0}\|u\|^{2}+\lambda \int_{J} f(t, x, u) u=(L u, u)-\lambda(h, u) .
$$

By condition (c3) there is a number $r>0$ such that

$$
f(t, x, s) s \geq \varepsilon_{0} s^{2}
$$

for $|s| \geq r$. Thus there is a function $\gamma \in H$ with

$$
f(t, x, s) s \geq \varepsilon_{0} s^{2}-\gamma(t, x)
$$

for all $(t, x, s) \in J \times \mathbf{R}$. From (3.8) we see that

$$
(1-\lambda) \varepsilon_{0}\|u\|^{2}+\lambda \int_{J} \varepsilon_{0}|u|^{2} d t d x-\int_{J} \gamma d t d x \leq|(L u, u)|+\|h\| \cdot\|u\| .
$$

Thus there is a constant $C$ with

$$
\begin{aligned}
\varepsilon_{0}\|u\|^{2} & \leq\left|\left(L u_{1}, u_{1}\right)\right|+\|h\| \cdot\|u\|+C \\
& \leq\left|L u_{1}\right|_{L^{1}} \cdot\left|u_{1}\right|_{L^{\infty}}+\|h\| \cdot\|u\|+C .
\end{aligned}
$$

By (3.6) and (3.7) we now obtain

$$
\varepsilon_{0}\|u\|^{2} \leq\left[\left(2 \varepsilon_{0}+\alpha\right) C_{4}\|u\|+C_{1}\right]\left[\left(2 \varepsilon_{0}+\alpha\right) C_{2}\|u\|+C_{3}\right]+\|h\| \cdot\|u\|+C
$$

and, hence,

$$
\varepsilon_{0}\|u\|^{2} \leq\left(2 \varepsilon_{0}+\alpha\right)^{2} k_{1}\|u\|^{2}+k_{2}\|u\|+k_{3}
$$

for some constants $k_{1}, k_{2}$, and $k_{3}$.

A subtraction yields

$$
\left[\varepsilon_{0}-\left(2 \varepsilon_{0}+\alpha\right)^{2} k_{1}\right]\|u\|^{2} \leq k_{2}\|u\|+k_{3} .
$$

By now choosing $\varepsilon_{0}$ and $\alpha_{0}$ sufficiently small we can insure that, since $\alpha<\alpha_{0}$,

$$
\varepsilon_{0}-\left(2 \varepsilon_{0}+\alpha\right)^{2} k_{1}>0,
$$

which, by (3.9), implies $\|u\|<M$ for some constant $M>0$. All possible solutions of (3.2) are thus bounded independently of $\lambda \in] 0,1$ [, and (3.1) has a solution.

PROOF OF THE COROLLARY. We take

$$
f(t, x, s)=-\alpha_{-} s^{-}+\alpha_{+} s^{+}+g(t, x, s) .
$$


By hypothesis $f$ is monotone nondecreasing in $s$. Also

$$
\begin{aligned}
|f(t, x, s)| & \leq \alpha_{-} s^{-}+\alpha_{+} s^{+}+|g(t, x, s)| \\
& \leq f(t, x, s)+2 \alpha_{-} s^{-}+2|g(t, x, s)| .
\end{aligned}
$$

By hypothesis, for each $\varepsilon>0$ there exists $\gamma_{\varepsilon} \in H$ with $|g(t, x, s)| \leq \varepsilon|s|+\gamma_{\varepsilon}(t, x)$. It follows that

$$
|f(t, x, s)| \leq f(t, x, s)+\left(2 \alpha_{-}+2 \varepsilon\right)|s|+2 \gamma_{\varepsilon}(t, x),
$$

which shows (c2) holds. Since $\varepsilon>0$ may be chosen arbitrarily small we can insure that $2 \alpha_{-}+2 \varepsilon<\alpha_{0}$, where $\alpha_{0}$ is the number in Theorem 1 , by requiring $\alpha_{-}<\alpha_{0} / 2$ and then choosing $\varepsilon$. Since

$$
\varliminf_{|s| \rightarrow \infty} s^{-1} f(t, x, s) \geq \min \left(\alpha_{-}, \alpha_{+}\right)>0,
$$

the corollary follows.

REMARK 5 . Instead of looking for solutions $2 \pi$-periodic in $t$ and $x$ we could also formulate our results for solutions $\omega_{1}$-periodic in $t$ and $\omega_{2}$-periodic in $x$ if we insist that $\omega_{1} / \omega_{2}$ be a rational number. This would insure that the d'Alembertian with these boundary conditions is realized in $H$ by a selfadjoint operator having properties like those of $L$ above. If $\omega_{1} / \omega_{2}$ is irrational, small divisors appear in the right inverse of $L$ which lead to unsolved difficulties.

4. A counterexample. It is easy to see that the corollary is false if $\alpha_{-}=0$ and $\alpha_{+}>0$. For example, if $g \equiv 0$ we have

$$
L u-\alpha^{+} u^{+}=h,
$$

and by taking inner products with 1 we see that $h$ must satisfy $(h, 1) \leq 0$. Thus (4.1) cannot be solvable for all $h \in H$. In spite of this, (4.1) is certainly solvable for some $h \in H$. One might expect a solution if

$$
(h, 1)=\int_{J} h d t d x<0 .
$$

We show however that there may not be a solution even then.

It is easy to show that $u \in \operatorname{ker} L$ if and only if $u=p(t+x)+q(t-x)$ for some $p, q$ each $2 \pi$-periodic on $\mathbf{R}$ with $p, q \in L^{2}(0,2 \pi)$.

Let $0<\delta_{1}<\delta_{2}<\pi$ and $p:[0,2 \pi] \rightarrow R$ be at least $C^{2}$ smooth and defined by

$$
p(s)=\left\{\begin{array}{l}
3 \text { if }|\pi-s| \leq \delta_{1}, \\
0 \text { if }|\pi-s| \geq \delta_{2} \text { and } 0 \leq s \leq 2 \pi \\
0 \leq p(s) \leq 3 \text { elsewhere. }
\end{array}\right.
$$

Extend $p 2 \pi$-periodically to all of $\mathbf{R}$ and define $\phi \in H$ by

$$
\phi(t, x)=p(t+x) \quad \text { for }(t, x) \in J .
$$

Then $\phi \in \operatorname{ker} L$; indeed, $\phi$ is a smooth (classical) solution of $u_{t t}-u_{x x}=0$ and $\phi$ is $2 \pi$-periodic in each of $x$ and $t$.

By choosing $\delta_{2}$ sufficiently small we can insure that

$$
\int_{J} \phi d t d x<4 \pi^{2} \text {. }
$$


On the other hand, by choosing $\delta_{1}$ sufficiently close to $\delta_{2}$, one can insure that

$$
\int_{J} \phi d t d x<\int_{J} \phi^{2} d t d x
$$

Now consider (4.1) with $h=\phi-1$. We observe that

$$
\int_{J} h d t d x=\int_{J} \phi d t d x-4 \pi^{2}<0 .
$$

Suppose $u \in D(L)$ solves

$$
L u-\alpha_{+} u^{+}=\phi-1 .
$$

Taking inner products with $\phi$ we obtain, since $(L u, \phi)=0$,

$$
0 \geq-\alpha_{+} \int_{J} u^{+} \phi d t d x=\int_{J}(\phi-1) \phi d t d x>0 .
$$

Thus (4.2) cannot have a solution.

If we now let $h=\mu(\phi-1)$ with $\mu \gg 1$ we see that there still is not a solution even when $-\int_{J} h$ is large.

\section{REFERENCES}

[C-H] S. N. Chow and J. K. Hale, Methods of bifurcation theory, Springer, Berlin and New York, 1982.

[L] H. Lovicarova, Periodic solutions of a weakly nonlinear wave equation in one dimension, Czechoslovak. Math. J. 19 (1969), 324-342.

[M.1] J. Mawhin, Periodic solutions of nonlinear dispersive wave equations, Constructive Methods for Nonlinear Boundary Value Problems and Nonlinear Oscillations (Albrecht, Collatz and Kirchgässner, eds.), Birkhäuser, Basel, 1979, pp. 102-109.

[M.2] _ Compacité, monotonie et convexité dans l'etude de problèmes aux limites semi-linéaires, Séminaire d'Analyse Moderne, No. 19, Univ. de Sherbrooke, 1981.

[M-W.1] J. Mawhin and J. Ward, Asymptotic nonuniform non-resonance conditions in the periodic Dirichlet problem for semi-linear wave equations, J. Math. Pures Appl. (to appear).

[M-W.2] _ Nonuniform non-resonance conditions in the periodic-Dirichlet problem for semi-linear wave equations with jumping nonlinearities (to appear).

[Wi] M. Willem, Periodic solutions of wave equations with jumping nonlinearities, J. Differential Equations 36 (1980), 20-27.

Department of Mathematics, University of Alabama, University, Alabama 35486 